Promoting and Nurturing Interactions with Open Access Books: Strategies for Publishers and Authors

\title{
Part 3: Recommendations, Guidelines, and Best Practices
}

Janneke Adema, Tobias Steiner

Community-led Open Publication Infrastructures for Monographs (COPIM)

Published on: Oct 21, 2021

License: Creative Commons Attribution 4.0 International License (C-BY 4.0). 
In this section we have collected different guidelines, best practices, and recommendations for publishers and authors who want to promote further interaction with their open access book(s) or book collections and wish to implement or experiment with (libre) open practices. These best practices are based on the research we have conducted for part 1 and $\underline{2}$ of this report and also draw extensively on three studies that have previously formulated in depth (structured) guidelines on open annotation and open peer review experiments and implementations. We can really recommend checking out these publications in more detail when introducing new interactive elements into your research or publishing workflows:

- Dandieu, C. and HIRMEOS Consortium (2019) Report on Post-Publication Open Peer Review Experiment. https://zenodo.org/record/3275651

- Fitzpatrick, K. and Rowe, K. (2010) 'Keywords for Open Review'. LOGOS: The. Journal of the World Book Community 21 (3-4), 133-141.

- Ross-Hellauer, T. and Görögh, E. (2019) 'Guidelines for Open Peer Review Implementation'. Research Integrity and Peer Review 4 (1), 4.

In this section of the report, we won't make a distinction between recommendations for authors and recommendations for publishers, partly in recognition that copyright (and hence rights to determine reuse levels) might lie with either party. Similarly, the distinctions between the researcher role and the publisher role, as earlier identified, can often become blurred in more experimental forms of publishing (Adema et al., 2021). Especially when we consider how scholars who run scholar-led presses (one of the main groups of presses we are writing this report for) are often themselves both authors and publishers. Instead we have divided the recommendations into three parts, broadly mirroring the implementation stages for a new open practice:

- Guidelines to implement or adopt a tool, technology, or open practice to promote interaction

- Guidelines to design a workflow that accommodates your open practice.

- Guidelines to engage communities in your open practice

\section{Guidelines to implement or adopt a tool, technology, or open practice to promote interaction}

As Fitzpatrick and Rowe have argued, when implementing a new tool into your workflow, "begin with your goals and core values, and choose the tools that support 
them, not the other way around" (Fitzpatrick \& Rowe,2010). From there, assess specific affordances and the technological feasibility of different tools as well as the technical capabilities of your own publishing systems or workflows to be configured for (more) open elements.

- Consider using open licenses on your book(s) or collections (for example Creative Commons or Traditional Knowledge licenses) that clearly indicate if and how your book(s) or collections can be reused or remixed and by who. Our Licensing_ section will have more details on a variety of implementation options. One particular starting point we'd like to recommend here is the OAPEN-UK Guide to Creative Commons for Humanities and Social Science monograph authors.

- Consider using openly-licensed content when working with third-party elements as part of your publishing project. This will not only help with future reuse, but also make things easier for your publishing process, as this will help to steer clear of potential issues with third-party rights to images (see Part 2: Licensing for more details).

- Consider enabling annotation on your book(s) or collections, for example by integrating the hypothes.is plugin on your website/platform or collection/server, by selecting platforms that already have hypothes.is integrated with their workflows, or by publishing your books on an (open source) platform that accommodates build-in annotations.

- Consider conducting an experiment with open peer review or remix and reuse on your book(s) or book collections.

- Consider enabling versioning on your book(s) or collections, for example by publishing your books on an (open source) platform that accommodates versioning.

- Assess the costs of various options/software/systems, looking at implementation and development costs (staff and resources), and future sustainability (including preservation).

- With a particular focus on preservation of publications that leverage some of the platforms discussed in Part 2, make sure to consider the recently-published report

"Guidelines for Preserving_New Forms of Scholarship" by the Mellon Foundation-funded project Enhancing Services to Preserve New Forms of Scholarship, led by New York University Libraries. 
- Consider potential barriers that readers might perceive when they try to interact with your publication on your chosen platform, such as log-in or registration barriers to comment, annotate, or reuse. Also consider questions of accessibility regarding screenreaders readability, alt texts for images, etc.

- Consider the longer-term sustainability implications of choosing a given tool or platform. Will the platform be kept up and running over longer periods of time? How likely is the chance that a commercial provider of your platform will later decide to hide your book behind a paywall or login wall to generate more revenue (see e.g., Academia.edu); Is your book exportable to a alternative open, reuseamenable formats (also important with respect to accessibility and preservation)?

\section{Guidelines to design a workflow that accommodates your open practice}

Similar to the first set of guidelines, the first thing you might want to consider is what you would like to achieve by promoting interactions around your book(s): what are your goals and values (e.g., improve peer review processes, stimulate conversations around books, promote their uptake and reuse) and how can you develop a technical and editorial workflow that reflects and supports those? What do you want to achieve and how does this line up with your values? You might want to consider setting priorities and implementing a phased approach prioritising the areas you would like to change most. Make sure to involve your colleagues and communities throughout when redesigning your workflows.

- Try to be flexible and consider identifying workflows, processes, and open practices suited to the book(s) or collections and the scholarly community in question.

- When setting up (new) open practices to promote interaction, be sure to be involved as moderators and facilitators within these kinds of experiments throughout (or identify others to do so).

- Try to provide support and detailed instructions on any of the (new) open practices, workflows, or platforms that you adopt and overcommunicate these (i.e., communicate them more often and in more settings than you think you should) to the communities involved in your experiment to ensure they can familiarise themselves with any new practice or technology and are engaged with the project and fully understand it. 
- Consider co-developing guidelines and code of conducts or regulatory systems that also clearly outline the values, goals, and aims of your publishing project, in order to assist with the above.

- At the same time, consider the editorial and authorial workload when (newly) introducing and conducting open practices, including designing and implementing a new workflow, communication tasks (including creating guidelines), consultations, screening, and monitoring. Ensure the labour involved for all participants remains sustainable over time.

- Consider preservation and sustainability early-on as part of your workflow. How will your annotations, open peer review reports, remixes, versions, or other forms of social engagement around books remain available in the longer term? How can these best be archived, what needs to be archived, and who needs to be involved in this process?

- Consider offering anonymity as an option for participants in an open forum (for example for early-career scholars).

- Evaluate your adoption of (new) open practices. Consider that setting up new open experiments takes time and you might not get the intended uptake the first time around. Be pragmatic and see how the results line up with your values, aims, and goals and adjust your practices or experiments where needed.

\section{Guidelines to engage communities in your open practice}

- Try to be conscious of, and sensitive to, community customs and differences where it comes to open practices. Make sure to consult with your communities about their preferences, needs and attitudes, and what open practices they feel comfortable with and which ones they are not.

- Try to explore beforehand how to (best) generate critical mass and engage communities around an open practice or experiment (for example in the case of open annotation or open peer review) before it is implemented. Think through how participants can be best mobilised and which fields and communities to target and engage.

- Try and set-up advanced commitments from participants to kick-start a project and find incentive factors (e.g., a workshop or conference) to mobilise participants and make use of social media to promote your open experiment where needed.. 
- Where possible and appropriate, involve your communities (for example authors, editors, editorial boards, reviewers, scholars) in developing workflows, protocols, and guidelines, or survey their opinions.

- Consider making some open practices optional, which might be especially important for so perceived 'controversial' practices. Opt-out (after consultation and overcommunication) might sometimes work better here than opt-in though to encourage participation.

- Explore ways to establish connections between books (e.g., in your collection) through hyperlinking or annotations for example, but also by making sure your books are published in machine-readable formats and with enriched metadata to encourage automated discovery.

- Explore ways to stimulate interactions with books on social media, for example by using hashtags.

- Explore ways how to more actively present your book or collections in ways that colleagues from different fields and members of the general public can find and engage them.

- Consider running pilots or experiments first of perceived controversial open practices.

Header image: Photo by The Public Domain Review on Flickr. Public Domain Mark 1.0 .

\section{Citations}

1. Adema, J., Mars, M., \& Steiner, T. (2021). Books Contain Multitudes: Exploring Experimental Publishing (1st ed.). PubPub. https://doi.org/10.21428/785a6451.933fa904

2. Fitzpatrick, K., \& Rowe, K. (2010). Keywords for Open Review. LOGOS: The. Journal of the World Book Community, 21(3-4), 133-141. 